\title{
Rails and anchors: guiding and trapping droplet microreactors in two dimensions $\dagger$
}

\author{
Paul Abbyad, $\oint^{a b}$ Rémi Dangla, $\oint^{a}$ Antigoni Alexandrou ${ }^{b}$ and Charles N. Baroud ${ }^{* b}$
}

This paper presents a method to control the motion of nanolitre drops in a wide and thin microchannel, by etching fine patterns into the channel's top surface. Such control is possible for drops that are squeezed by the channel roof, by allowing them to reduce their surface energy as they enter into a local depression. The resulting gain in surface energy pulls a drop into the groove such that localized holes can be used as anchors for holding drops, while linear patterns can be used as rails to guide them along complex trajectories. An anchored drop can remain stationary indefinitely, as long as the driving flow rate is below a critical value which depends on the hole and drop sizes. By micro-fabricating holes into a grid pattern, drops can be arrayed and held in the observation field of a microscope against the mean carrier flow. Their contents can then be modulated by gas exchange with the flowing carrier oil. We demonstrate in particular how the $\mathrm{pH}$ or the oxygen levels within the drops can be controlled spatially and temporally, either by exposing rows of drops to two streams of oil at different gas concentrations or by periodically switching oil inputs to vary the gas concentration of drops as a function of time. Oxygen control is used to selectively deoxygenate droplets that encapsulate red blood cells from patients suffering from sickle cell disease, in order to study the polymerization of intracellular hemoglobin. Cycles of oxygenation and deoxygenation of anchored droplets induce depolymerization and polymerization of the hemoglobin, thus providing a method to simulate the cycling that takes place in physiological flows.

\section{Introduction}

Performing a large number of experiments in parallel has become increasingly important in molecular and cell biology. The most common laboratory tool for such parallelization is the multi-well plate, in which different reactions can take place in a large grid of independent wells. Experiments in such two-dimensional arrays simplify the observation, manipulation, and analysis of large data sets. For this reason, many emerging miniature devices aimed at replacing standard laboratory equipment have explored ways to organize experiments in an array format, for example in DNA-chips and cellular arrays. ${ }^{1,2}$

Parallelization within the field of microfluidics is of great interest and continues to be explored through different approaches. For instance, the early work on collapsible channels led to the large-scale integration of microfluidic networks, in which isolated chambers were used to perform independent reactions. ${ }^{3}$ Other approaches involve for example a large number of independent microchannels that are replicated side by side, in view of integrating them with standard micro-pipette instruments. ${ }^{4}$ Droplet microfluidics is particularly attractive for

${ }^{a}$ Laboratoire d'Hydrodynamique (LadHyX) and Department of Mechanics, Ecole Polytechnique, CNRS, 91128 Palaiseau, France

${ }^{b}$ Laboratoire d'Optique et Biosciences (LOB), Ecole Polytechnique, INSERM U696, CNRS, 91128 Palaiseau, France. E-mail: baroud@, ladhyx.polytechnique. $\mathrm{fr}$

$\dagger$ Published as part of a LOC themed issue dedicated to French Research: Guest Editor Professor Jean-Louis Viovy.

$\S$ These authors contributed equally to this work. parallel experiments as the content of each drop is isolated and controlled. Furthermore, individual droplets can be transported, sorted, merged and divided. ${ }^{5-9}$ However, arraying droplets within microchannels must rely on immobilizing them in a controlled fashion in the presence of a mean flow that constantly pushes them downstream. Different groups have proposed ways to hold droplets stationary, either using localized laser heating ${ }^{\mathbf{1 0 , 1 1}}$ or relying on microfabricated mechanical obstacles. ${ }^{12-16}$

In the case of mechanical traps, the devices rely on the drop's resistance to being squeezed. ${ }^{12-16}$ Indeed, an unconstrained drop takes on a spherical shape in order to minimize its surface energy, which is equal to its surface area times its surface tension. Therefore, if a drop is placed in a region of low surface energy, it will resist leaving into a region where it will be squeezed. The resistance force is given by the gradient of the surface energy. This can be applied to block a droplet either by placing it in a pocket and pushing it with the mean flow against a small exit hole $\mathrm{e}^{13,14,16}$ or by allowing it to expand into a chamber of increased dimensions compared to its surroundings. ${ }^{12,15}$ In all of the above cases, the drops were positioned in an array to maximize the number of drops in the microscope field of view.

However, holding a droplet can be performed using much weaker constraints than the ones described in the literature. As we detail below, flattened drops that are constrained by the top and bottom boundaries of the microchannel can be manipulated by imposing modulations of the channel height, e.g. by etching a pattern of holes and grooves into one of the microchannel surfaces. The technique that we present here relies on simple multi-layer photolithography, which allows any pattern to be drawn on the channel roof. In particular, a circular hole anchors a drop in place (Fig. 1A and B) and a series of patterned holes is 
A

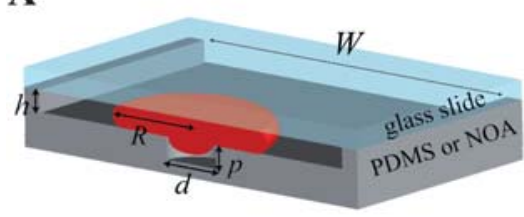

C

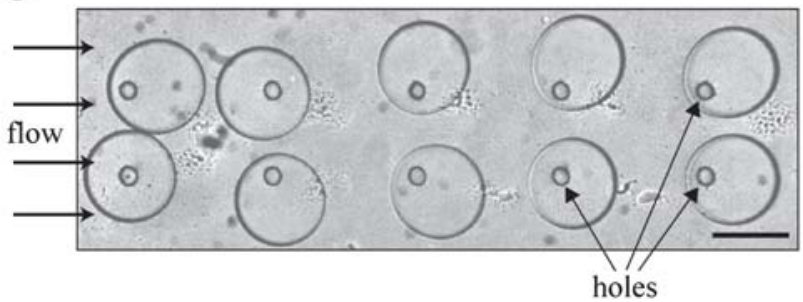

Fig. 1 (A) Sketch of the experimental device which defines the geometric parameters. (B) A water drop anchored to a hole in the channel roof with oil flowing left to right. (C) An array of anchored droplets. The scale bar represents $250 \mu \mathrm{m}$ in all images.

used to obtain a droplet array (Fig. 1C and Movie S1). Furthermore, linear grooves are used to guide drops in twodimensions (2D) much like a rail is used to guide a train. The combination of rails and anchors therefore provides truly $2 \mathrm{D}$ manipulation of drops, far from the lateral walls of the microchannel and with minimal interactions between the drops. The simplicity of this approach ensures its robustness, since it relies on a balance between two well-understood physical mechanisms. Finally, the drops that are anchored in this way keep a large exchange area with the flowing external oil phase, which allows gas transfer between the oil phase and the stationary droplet as shown in Abbyad et al. ${ }^{17}$ This can be used to transport a continuous supply of oxygen for cell cultures in droplets or to provoke a temporal or spatial variation in gas content in a droplet array.

The body of the manuscript is divided into three parts. The Materials and methods section (Section II) explains the device fabrication and experimental protocol. The second part (Section III) discusses the physical principles and shows how droplets can be guided and anchored using etched patterns. It further quantifies the strength of an anchor point by measuring the critical flow rate of the carrier phase required to displace the drop from the anchor. In the third part (Section IV), the gas exchange with the carrier phase is used to impose variable conditions within an array of anchored drops, both in space, across different rows of the array, and in time. This work is motivated by the study of sickle-cell anemia and the gas-exchange techniques are used to induce red blood cell sickling in anchored droplets as shown in Section IVB.

\section{Materials and methods}

\section{A. Microfluidic device fabrication}

Chips of two different materials were used for the experiments. PDMS devices were used for the experiments on anchor strength and rails (Section III). For experiments involving gas exchange (Section IV), chips were made of gas impermeable Norland
Optical Adhesive 81 (NOA81). The fabrication procedures for both PDMS and NOA81 devices are explained briefly below.

Microfluidic devices with channel depth modulations were fabricated using the dry film photoresist soft lithography technique described by Stephan et al. ${ }^{18}$ since it enables rapid prototyping of multi-level structures. Here, the masters were etched in Eternal Laminar E8013 and Eternal Laminar E8020 negative films of thickness $33 \pm 2 \mu \mathrm{m}$ and $49 \pm 2 \mu \mathrm{m}$ respectively.

The fabrication procedure is as follows: (i) photoresist layers were successively laminated onto a clean glass slide using an office laminator at $100{ }^{\circ} \mathrm{C}$ until the desired height $h$ of the main channel was reached. (ii) The photoresist film was exposed to UV through the photomask of the main network (test section, entrance and exit channels). (iii) Additional photoresist layers were laminated on top of the exposed film until the desired depth $p$ of the anchors (or rails) was reached. (iv) The stack of photoresist films was again exposed to UV, through the photomask of the depth modulations aligned with the previous network. (v) Finally the full structure was developed by immersion in an aqueous bath of carbonate potassium at $1 \%$ mass concentration. PDMS (Dow Corning SYLGARD 184, 1/10 ratio of curing agent to bulk material) was then poured over the master and cured $\left(2\right.$ hours at $\left.70^{\circ} \mathrm{C}\right)$. The PDMS device was sealed on a glass slide by plasma bonding to obtain a microchannel as sketched in Fig. 1.

Devices used for oil-droplet gas exchange were made of gasimpermeable photocurable glue, NOA, following the protocol described in Bartolo et al. ${ }^{19}$ Briefly, a PDMS mold was made from the master as described above. This PDMS mold was in turn used as a template to produce a PDMS stamp. Exposing the PDMS mold to gaseous $1 \mathrm{H}, 1 \mathrm{H}, 2 \mathrm{H}, 2 \mathrm{H}$-perfluorodecyltrichlorosilane (Alfa Aesar) for 3 hours in a sealed container ensured that the PDMS layers did not permanently bond during the curing process. The PDMS stamp was then pressed over a drop of Norland Optical Adhesive 81 (NOA81, Thorlabs) on a glass coverslide before exposure to a UV lamp (exposure for 40 seconds at a power of $7 \mathrm{~mW} \mathrm{~cm} \mathrm{~cm}^{-2}$ ). A thin layer of glue remained uncured on the surface due to the presence of oxygen, which allowed the device to be sealed with a glass slide with a second exposure to a UV source (exposure for 40 seconds at a power of 7 $\mathrm{mW} \mathrm{cm}{ }^{-2}$ ). To render the internal channel surface hydrophobic, a glass treatment chemical (Aquapel, PPG Industries) or $1 H, 1 H, 2 H, 2 H$-perfluorodecyltrichlorosilane $(20 \mu \mathrm{L}$ in $1 \mathrm{~mL}$ of FC-40) was flowed briefly through the microchannel.

\section{B. Oxygen control}

To vary its oxygen concentration, the oil was flowed through approximately $50 \mathrm{~cm}$ of gas permeable silicone tubing $(0.3 \mathrm{~mm}$ internal diameter and $0.6 \mathrm{~mm}$ outer diameter, Helix) in a vial with a controlled oxygen atmosphere prior to entering the NOA microfluidic device. By varying the relative flow rates of nitrogen gas and air into the vial, the oxygen concentration in the vial could be adjusted from $0 \%$ to $21 \%$ oxygen (atmospheric oxygen percentage) i.e. from 0 to $21 \mathrm{kPa}$ oxygen partial pressure $\left(p \mathrm{O}_{2}\right)$. The flowing fluids equilibrated with the oxygen partial pressure in the vials through gas exchange across the silicone tubing. After the gas exchange vial, it is important to maintain a virtually gastight environment. Therefore low-gas permeability tubing made 
of polyether ether ketone (PEEK, Upchurch) was used to connect the vial to the NanoPort (Upchurch) inlets of the microfluidic device.

\section{Determination of on-chip oxygen concentration}

The on-chip oxygen concentration in individual trapped microdroplets was determined using a fluorescence probe, ruthenium tris(dipyridyl) dichloride hexahydrate (RTDP) (Sigma-Aldrich), using a setup recently described in Abbyad et al. ${ }^{17}$ The fluorescence lifetime of the probe is proportional to the oxygen partial pressure and varies from $600 \mathrm{~ns}$ for $0 \mathrm{kPa}$ oxygen to $380 \mathrm{~ns}$ for 21 kPa oxygen.

In contrast with our earlier study on flowing droplets, a slow drift to shorter lifetimes was observed when measurements were made on an anchored droplet over extended periods. The drift was not observed in the absence of excitation light or for a flowing stream of RTDP solution. Therefore, this drift is likely associated with production of an unknown photoproduct with a shorter lifetime that accumulates in the small volume of a droplet. Within the timescale of the experiments, the drift in lifetime was constant in time and was more substantial for smaller droplets. For a droplet with a diameter of $320 \mu \mathrm{m}$, the change in lifetime was $0.7 \mathrm{~ns}$ per second of illumination with excitation light. To minimize this drift, the excitation light was reduced to 0.5 second exposures every 2 seconds (for fast oxygen changes as in Fig. 5D) or every 20 seconds (for slow oxygen changes as in Fig. 5C). In the treatment of the lifetime data, a linear slope corresponding to the drift was subtracted to correct for the effect.

\section{D. pH control}

The $\mathrm{pH}$ of anchored droplets was altered by gas exchange with $\mathrm{CO}_{2}$ dissolved in the carrier oil. To monitor the droplet $\mathrm{pH}$, the indicator, bromothymol blue (Sigma-Aldrich), was dissolved in the aqueous phase $\left(0.5 \mathrm{mg} \mathrm{mL}^{-1}\right)$ and the $\mathrm{pH}$ of the solution was adjusted to about 9 using sodium hydroxide. To dissolve $\mathrm{CO}_{2}$ in the carrier oil, a solution of FC-40 with surfactant was placed for 30 minutes in an incubator with a controlled atmosphere containing $5 \mathrm{kPa} \mathrm{CO}_{2}$. The solution was then transferred to a gastight syringe (SGE).

\section{E. Sickling of red blood cells}

Blood samples were obtained from untreated sickle cell patients (Tenon Hospital, Paris) and stored at $4{ }^{\circ} \mathrm{C}$ using sodium citrate as an anti-coagulant. Samples were used within a week after extraction. Red blood cells were washed three times with PBS buffer (137 mM NaCl, $2.7 \mathrm{mM} \mathrm{KCl}, 8.0 \mathrm{mM} \mathrm{Na} 2 \mathrm{HPO}_{4}, 1.5 \mathrm{mM}$ $\mathrm{KH}_{2} \mathrm{PO}_{4}, 5.5 \mathrm{mM}$ glucose, $2 \mathrm{mM} \mathrm{CaCl}, \mathrm{pH}$ 7.4) by sequential dilution and centrifugation $(3000 \mathrm{~g})$. For experiments, the washed red blood cells were diluted 100-200 fold in D-PBS buffer (137 $\mathrm{mM} \mathrm{NaCl}, 2.7 \mathrm{mM} \mathrm{KCl}, 8.0 \mathrm{mM} \mathrm{Na} \mathrm{HPO}_{4}, 1.5 \mathrm{mM} \mathrm{KH_{2 }} \mathrm{PO}_{4}$, $0.9 \mathrm{mM} \mathrm{CaCl}_{2}, 0.5 \mathrm{mM} \mathrm{MgCl}_{2}, \mathrm{pH} 7.4$, Invitrogen) and $35 \% \mathrm{v} / \mathrm{v}$ Optiprep (Sigma) to prevent cell sedimentation. Biocompatible surfactant, $\quad 0.5 \%$ dimorpholinophosphate-PFPE (DMPPFPE $^{20}$ in FC-40, was added to the oil. The microfluidic chip was heated to $30{ }^{\circ} \mathrm{C}$ using a heater plate, thus approaching physiological temperatures.
Polarization microscopy was used to detect intracellular hemoglobin fibers in red blood cells. Images were obtained with two crossed thin-film linear polarizers placed in the optical path of the microscope before and after the sample as described in Abbyad et al. ${ }^{17}$

\section{Droplet anchoring by depth modulations}

\section{A. Physical background}

The basic geometry of the test section is a thin microchannel of height $h$ much smaller than its width $W$ and length $L$, referred to as a Hele-Shaw cell in the fluid mechanics literature. ${ }^{21}$ It is filled with a fully wetting oil, in which case a thin film of oil prevents the water drops from being in direct contact with the channel walls. In this geometry, large drops (of volume greater than the largest sphere that can be inscribed in the channel $V_{\mathrm{c}}=4 \pi / 3(\mathrm{~h} /$ $2)^{3}$ ) are constrained by the top and bottom walls of the channel such that they can only move in the plane of the Hele-Shaw cell. $V_{\mathrm{c}}$ is equal to $0.5 \mathrm{~nL}$ for a 100 micron high channel and typical droplet volumes used here were around $10 \mathrm{~nL}$. Such drops adopt a flattened pancake shape, i.e. a rounded cylinder of radius $R$ and height $h$, as sketched in Fig. 1A.

A consequence of the vertical confinement on the drops is that they become sensitive to depth modulations of the microchannel. Indeed, any interface between two fluids has a surface energy $\varepsilon_{\gamma}$, defined as the product between interfacial tension $\gamma$ and the surface area of the interface. For a drop of constant volume, this surface energy is minimal for a spherical shape and it increases as the drop flattens into a pancake shape. Therefore, in a microchannel of non-uniform height, flattened droplets are captured by regions of reduced confinement which lower their surface energy.

By this mechanism, a hole, i.e. a circular region of increased channel height of diameter $d$ and depth $p$, is able to trap a droplet. Indeed, when a droplet reaches the hole, it lowers its surface energy by partially entering into the cavity even if $d$ is much smaller than the radius of the drop $R$, as sketched in Fig. 1A. The drop deforms and is held only locally, as if it were anchored to the hole. In that sense, a hole can be seen as a surface energy well in which the drop is trapped, in an analogy with particle trapping in potential energy wells. An external force is therefore necessary for the drop to detach from the anchor site.

In the presence of an external flow, the droplet is pushed by the hydrodynamic drag of the carrier phase and it will remain anchored only if the strength of the trap is sufficient to balance the drag from the flow. The trap strength can therefore be quantified by measuring the critical flow rate of the carrier phase $Q_{\mathrm{c}}$ above which the hole is no longer able to hold the droplet.

\section{B. Determination of anchor strength}

We measured the anchor strength experimentally using a PDMS microfluidic chip that enabled separate control of the size of the drops and the advection flow velocity, as sketched in the inset of Fig. 2. The design consisted of a main test section, a Hele-Shaw cell of height $h=35 \mu \mathrm{m}$ or $h=50 \mu \mathrm{m}, W=3 \mathrm{~mm}$, and length $L=$ $6 \mathrm{~mm}$, which contained a single hole. It was connected upstream to a flow focusing device which produced water droplets in oil (FC-40 $+2 \%$ surfactant). The fluids were injected using syringe 


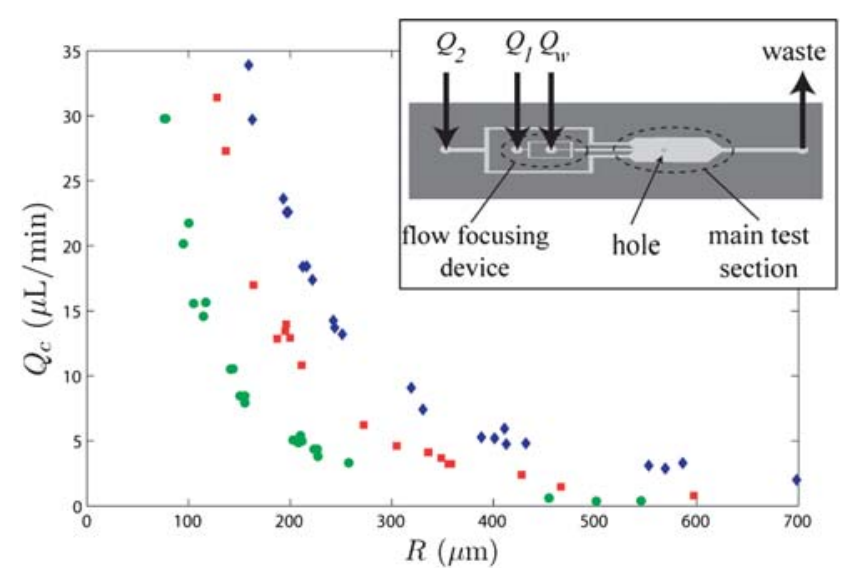

Fig. 2 Inset: sketch of the microfluidic chip featuring the flow focusing device for droplet production, the oil inlet for flow rate control and the Hele-Shaw cell with a single anchor for the measurement of the anchor strength. Main: critical flow rate $Q_{\mathrm{c}}$ as a function of droplet radius $R$ for three channels and anchor geometries: $h=35 \mu \mathrm{m}, d=50 \mu \mathrm{m}$ and $p=35$ $\mu \mathrm{m}$ (green circles); $h=50 \mu \mathrm{m}, d=75 \mu \mathrm{m}$ and $p=50 \mu \mathrm{m}$ (red squares); $h=$ $50 \mu \mathrm{m}, d=100 \mu \mathrm{m}$ and $p=50 \mu \mathrm{m}$ (blue diamonds).

pumps, at flow rates $Q_{\mathrm{w}}$ for water and $Q_{1}$ for the oil, which could be tuned to adjust the droplet size. A second oil inlet with a flow rate $Q_{2}$ also led to the test section. The total flow rate of the advecting oil in the test region was thus $Q_{\mathrm{o}}=Q_{1}+Q_{2}$.

Droplets of a given radius $R$ were first produced at the flow focuser ${ }^{22}$ and flowed into the Hele-Shaw cell, at which point the entrainment flow rate $Q_{\mathrm{o}}$ was lowered to a value at which a single droplet was trapped by the hole. At this stage the flow of water was stopped to end the production of droplets and all droplets, except for the anchored droplet of interest, were flushed out of the test section by the carrier oil. The value of $Q_{\text {o }}$ was then incrementally increased up to the critical value $Q_{\mathrm{c}}$ at which the anchored droplet was pushed out of the hole by the oil flow. For a given geometry of the test region $(h, d, p)$, this protocol was repeated for droplets of various radii in order to determine the dependence of $Q_{\mathrm{c}}$ on $R$.

The results for three channel and hole geometries $(h, d, p)$ are presented in Fig. 2. We observe that droplets are trapped by the holes at low flow rates and that they can be held in this stationary state indefinitely unless the flow rate is increased above a critical value $Q_{\text {c }}$. For a given hole geometry and droplet radius $R$, this threshold is well defined since fluctuations in successive measurements are below 5\%. The strength of anchors displays a general trend: the critical flow rate $Q_{\mathrm{c}}$ decreases with $R$ regardless of the channel and hole geometry. This trend is due to the increase in drag force with droplet radius $R \cdot{ }^{23}$ Furthermore, the experimental curves $Q_{\mathrm{c}}(R)$ for the three geometries do not intersect, indicating that anchors can be ranked according to their trapping strength, independent of drop radius $R$. For example, for a channel of height $h=50 \mu \mathrm{m}$, the hole of diameter $d=100 \mu \mathrm{m}$ is a stronger anchor than the hole with diameter $d=75 \mu \mathrm{m}$. This is in agreement with the intuitive argument that the larger the hole, the more the surface energy decreases as the droplet deforms into it.

From this empirical analysis, anchors appear as a simple and robust device for trapping droplets in an external flow. In addition, this technique is flexible and reversible since the flow rate can be tuned to below $Q_{\mathrm{c}}$ to anchor a droplet then increased above $Q_{\mathrm{c}}$ to flush it out after an experiment. Anchors are also omnidirectional, capable of anchoring a droplet regardless of the external flow direction.

\section{Droplet interactions}

In practical applications, multiple drops may be flowing inside the microchannel such that mobile droplets will collide with anchored ones. This raises the issue of droplet interactions which we explore here. Using the same microfluidic chip as in the previous section, we produced a continuous train of regularly sized droplets that flowed into the Hele-Shaw cell containing a single anchor. The total flow rate was adjusted by varying the secondary flow rate $Q_{2}$.

As expected, if the total flow rate $Q_{\mathrm{o}}$ exceeded the critical flow rate $Q_{\mathrm{c}}$, the droplets were not trapped by the anchor and they were regularly advected across the test section. However, within a range of flow rates below the threshold, the droplet train entered a buffering mode: a single droplet is trapped by the anchor until the following one in the train arrives, collides, and pushes it out of the hole, thus replacing it. This behavior is illustrated by the time sequence shown in Fig. 3A which shows droplets of radius $R=180 \mu \mathrm{m}$ flowing from left to right with an

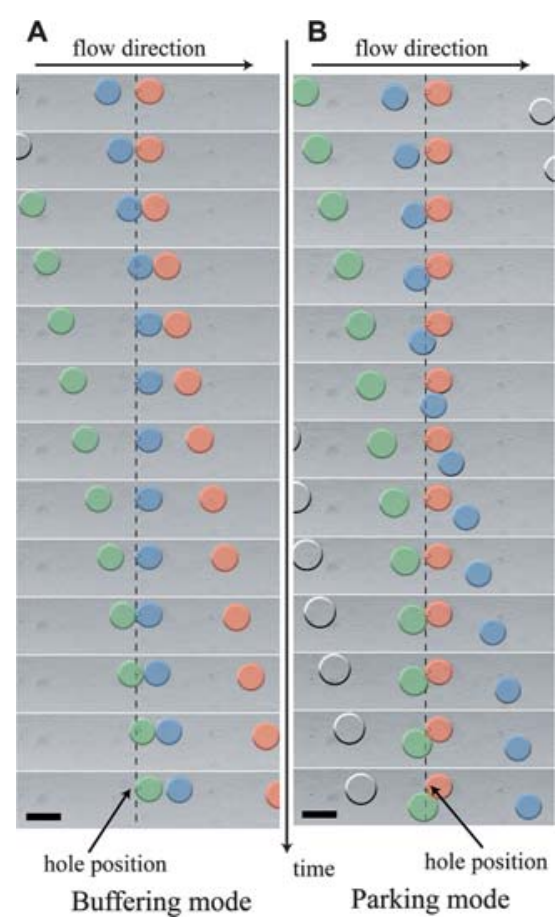

Fig. 3 Image sequences of a train of droplets of radius $R=180 \mu \mathrm{m}$ flowing from left to right in a Hele-Shaw cell of height $h=50 \mu \mathrm{m}$ over an anchor of diameter $d=75 \mu \mathrm{m}$. Droplets are artificially colored by image processing to facilitate tracking of individual droplets. (A) For $Q_{\mathrm{o}}=12$ $\mu \mathrm{L} \mathrm{min}{ }^{-1}$, the train is in buffering mode (sequence frame rate: 1 frame per second). Droplets enter the Hele-Shaw cell at a rate of 15 droplets per

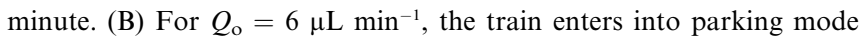
(sequence frame rate: 0.5 frames per second). Droplets enter the HeleShaw cell at a rate of 8 droplets per minute. The scale bars represent 500 $\mu \mathrm{m}$. 


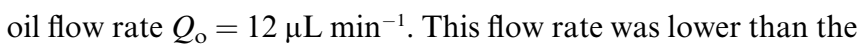

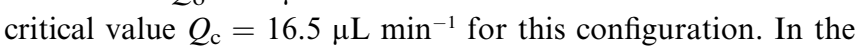
figure, the droplets are artificially colored by image processing to easily distinguish individual droplets. In the first 2 frames, the red drop is held stationary by the anchor when the blue drop arrives, advected by the external flow. The instant the two drops collide (frame 3), the red drop is pushed off the anchor and the blue drop takes its place. The blue drop is now stationary and anchored to the hole (frames 5-9) until the arrival of the next drop. This cycle can continue indefinitely, with each successive droplet replacing the previous droplet in the anchor (see Movie S2 ).

If we decrease the flow rate $Q_{\mathrm{o}}$ further, the buffering mode switches to a parking mode: a unique droplet is held in place by the anchor and remains anchored as later drops collide with it. This behavior is illustrated by the time sequence shown in Fig. 3B which has the same geometrical configuration as Fig. 3A but with

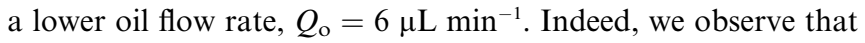
the red drop is held stationary by the anchor throughout the time sequence and resists collision with the incoming blue and green drops.

These modes illustrate the flexibility of the anchors as trapping devices as it is possible to switch from one mode to the other by varying the external flow rate. Applications are numerous: the buffering mode could be used to observe for a limited duration successive droplets at a fixed position, increasing the integration time for example in fluorescence experiments; the parking mode is useful to observe droplets for an extended period at fixed positions or to select a single droplet in a continuous train of droplets. This is of particular interest when studying a slow biological or chemical process in a droplet.

\section{Drops on rails}

Similar gain in surface energy is achieved when a pancake droplet encounters grooves that extend along one direction. In this case, however, the indentation only impedes movement along the direction perpendicular to the groove but not along the groove direction. In this way, a drop will be guided by the groove while being pushed by the external flow, as a train follows a rail. These rails can have much smaller widths than the radius of the droplet, as shown in Fig. 4A where a sinusoidal rail pattern guides droplets along its path. All the drops follow the path dictated by the micro-fabricated etch, although they do not necessarily remain centered on the rail, due to the drag force from the external oil flow. The velocity of the drops depends on the relative local orientation of the rail with respect to the direction of the mean oil flow. As seen in Movie S3, the drops flow rapidly along sections where the rail is oriented in the same direction as the mean flow but more slowly in sections where the rail is strongly inclined relative to the mean flow.

Again, the simple microfabrication procedure allows complex rail networks to be implemented, as shown in the snapshot of Fig. 4B. In this image, drops that are of similar size as the rail width are guided into a complex distribution along the channel width. In this example, droplet-droplet interactions within the crowded central rail push some drops to take alternative paths between the inlet and the exit. If a drop "derails", it will follow the mean flow until it reaches an available position in another rail. Drops spend variable amounts of time in the test section
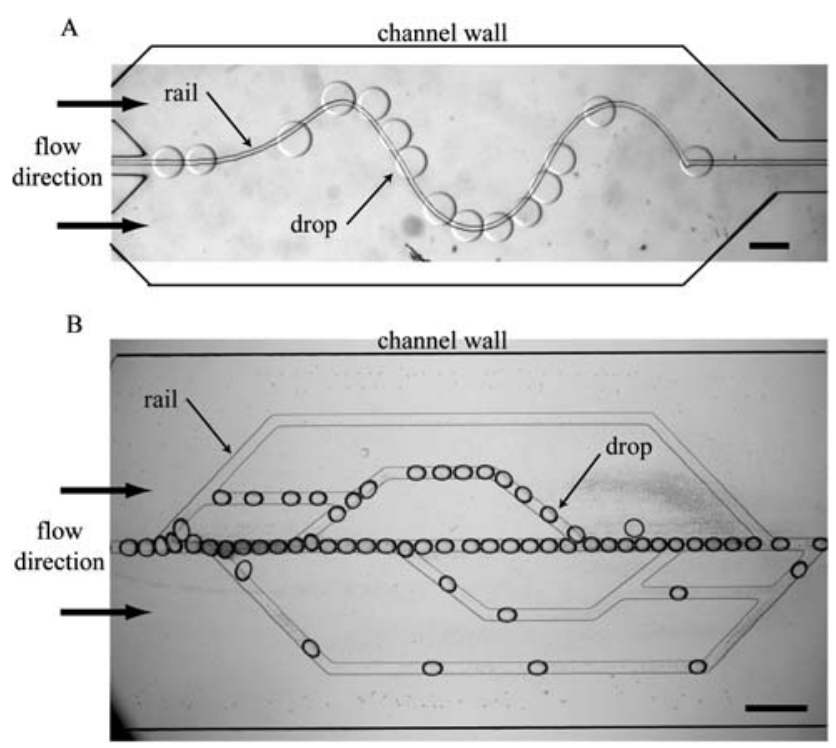

Fig. 4 Microscope images of multiple water drops guided from left to right along microfabricated rails (50 $\mu \mathrm{m}$ in width and $50 \mu \mathrm{m}$ in depth) in an external flow of fluorinated oil. (A) Large drops of radius $R \approx 200 \mu \mathrm{m}$ follow a narrow sinusoidal rail inside a Hele-Shaw cell of height $h=100$ $\mu \mathrm{m}$. (B) Smaller drops of radius $R \approx 50 \mu \mathrm{m}$ follow a complex network of rails with bifurcations and junctions inside a Hele-Shaw cell of height $h=$ $50 \mu \mathrm{m}$. The scale bars represent $500 \mu \mathrm{m}$.

since it takes longer to follow the off-centered paths as compared with the central rail.

These two examples of drops on rails demonstrate the basic functions of guiding drops in a 2D area. Drops will follow a micro-fabricated rail and this can be used to divide an initial sample into many parallel paths. Conversely, several input samples can be brought close together by designing drop inputs and rail patterns accordingly. Furthermore, the combination of rails and anchors provides a way to bring different initial samples into close proximity and to hold them in place, in order to facilitate their observation within a single image. Networks of interconnected rails can be used for complex operations by directing droplets at junctions. Droplets can be guided through a complex network of rails by passive means, for instance by relying on droplet-droplet interactions and rail geometry. An example of this crowding effect is visible in Fig. 4B, where some drops are pushed away from the central rail by their neighbors. Moreover, the guidance can also be determined actively by applying an external force that selects between two possible rails, for example through electric fields ${ }^{24}$ or laser heating. ${ }^{8}$ In the rest of the manuscript, we focus on arrays of stationary droplets and show how the oil flow can be used to control the drop contents.

\section{Spatial and temporal control in droplet arrays}

In two-phase microfluidic devices, the carrier oil serves both for the production and the transport of microdroplets. We have shown in a recent publication ${ }^{17}$ that the perfluorinated carrier oil can also be used as either a source or a sink for oxygen exchange in aqueous droplets. This approach can be generalized to many different gases due to their high solubility in perfluorinated oils. Below, we show how this gas exchange can be used to control the 
contents of anchored droplet arrays. For gas exchange applications, the devices are made of a gas impermeable chip material, NOA photocurable glue, rather than gas permeable PDMS, to ensure that exchange occurs solely between the oil and aqueous phases.

\section{A. Oil-droplet gas exchange}

A train of droplets was produced at a flow focuser and transported to an array of anchors as shown in Fig. 5A. By working at
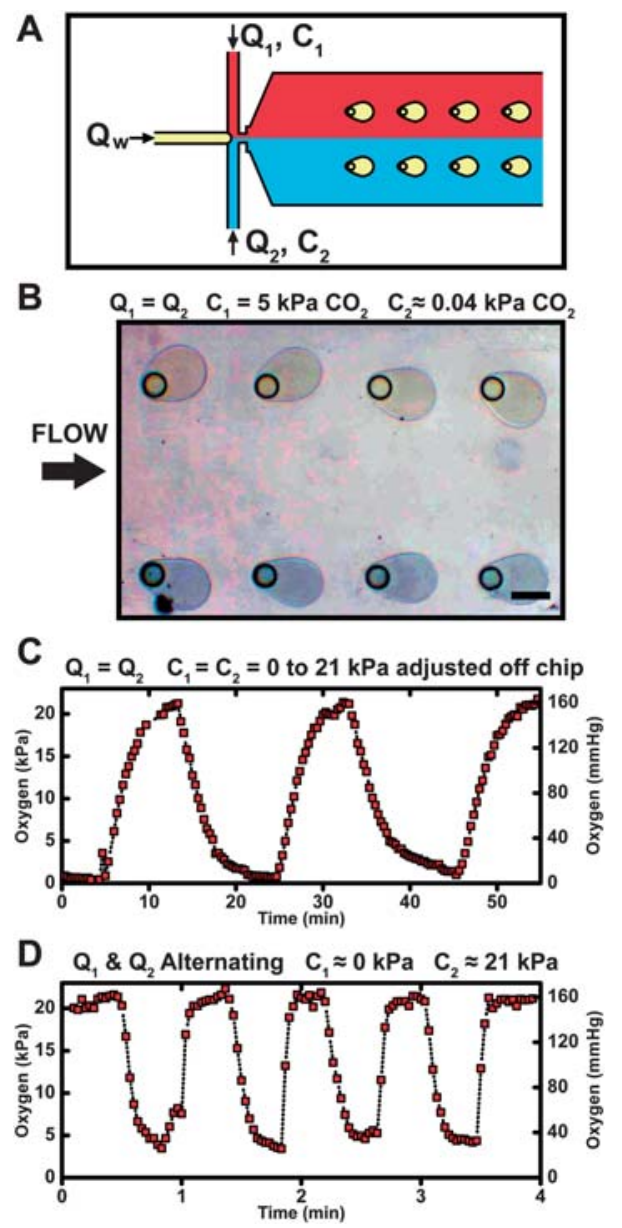

Fig. 5 (A) Schematic of the flows in the microfluidic chip for gas exchange. The flow rates and gas contents are annotated as follows: $Q_{\mathrm{w}}$ flow rate of aqueous phase, $Q_{1}$ flow rate of oil inlet one, $Q_{2}$ flow rate of oil inlet two, $C_{1}$ gas partial pressure of oil inlet one and $C_{2}$ gas partial pressure of oil inlet two. (B) Color image of trapped droplets containing the $\mathrm{pH}$ indicator bromothymol blue. The oil flow rates, $Q_{1}$ and $Q_{2}$, are both held constant at $2 \mu \mathrm{L} \mathrm{min}{ }^{-1}$. The upper oil stream, $C_{1}$, contains 5 $\mathrm{kPa}$ dissolved $\mathrm{CO}_{2}$ and the lower oil stream, $C_{2}$, contains ambient dissolved $\mathrm{CO}_{2}(0.04 \mathrm{kPa})$. The droplets exposed to the upper oil stream are yellow while those exposed to the lower oil stream are blue. The scale bar represents $200 \mu \mathrm{m}$. (C) Oxygen partial pressure $\left(p \mathrm{O}_{2}\right)$ of an anchored droplet as determined by RTDP fluorescence lifetime while alternating the oxygen concentration upstream of the chip. The partial pressure of oxygen is varied from $0 \mathrm{kPa}$ to $21 \mathrm{kPa}$. The flow rate is held constant at $Q_{1}=Q_{2}=1 \mu \mathrm{L} \mathrm{min}{ }^{-1}$. (D) Oxygen partial pressure $\left(p \mathrm{O}_{2}\right)$ of a trapped droplet while alternating the flows of the two oil channels. The partial pressures of oxygen of the oil channels are $C_{1}=21 \mathrm{kPa}$ and $C_{2} \approx 0 \mathrm{kPa}$.

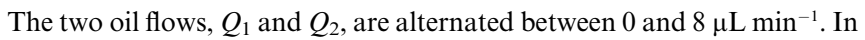
all cases, the height $h$ of the anchor is $50 \mu \mathrm{m}$ and its depth $p$ is $50 \mu \mathrm{m}$. flow rates in the parking mode range, anchored drops remained stationary despite the external flow of carrier oil. In this situation, the flowing oil can be used to control the gas content of the droplets while they remain in the observation area of the microscope. The control parameters of this experiment are the flow rates of the oil inputs, $Q_{1}$ and $Q_{2}$, as well as their respective gas partial pressures, $C_{1}$ and $C_{2}$. Controlling the flow rates and gas contents of the two incoming oil streams allows both spatial and temporal control of gas content in a droplet array.

For example, spatial variation of the dissolved gas content along the width of the channel is achieved by using two laminar oil streams at different dissolved gas concentrations. In our chip geometry, setting $Q_{1}=Q_{2}$ and $C_{1} \neq C_{2}$ results in the droplets in the upper part of the channel being exposed to a different gas partial pressure from those in the lower part as shown schematically in Fig. 5A. In the same microscope viewing area, the trapped droplets can thus be subjected to different gas conditions if advection dominates over diffusion. This situation corresponds to a large Peclet number $P e=U L / D$, where $U$ is the oil velocity, $L$ is the distance between two droplets, and $D$ is the diffusion coefficient of the gas molecules.

We demonstrate spatial control of the $\mathrm{pH}$ using two different concentrations of $\mathrm{CO}_{2}$ in the two streams: $C_{1}=5 \mathrm{kPa}$ and $C_{2}=$ $0.04 \mathrm{kPa}$ (ambient $\mathrm{CO}_{2}$ ). A pH indicator, bromothymol blue, was dissolved in the aqueous phase and the indicator undergoes a color change from blue to yellow as the $\mathrm{pH}$ changes from 8 to 6 . The initial $\mathrm{pH}$ of the aqueous phase was adjusted to 9 and therefore all droplets were blue as they were produced. The droplets were anchored in two rows, separated by $1 \mathrm{~mm}$, along the length of the channel. The droplets in the upper row were exposed to a higher concentration of $\mathrm{CO}_{2}$, which dissolves in the droplet as carbonic acid. In a few seconds, the dissolved acid decreased the droplet's $\mathrm{pH}$ from 9 to 5 with a corresponding droplet color change from blue to yellow (Fig. 5B). The drops of the lower row were exposed to a far lower concentration of $\mathrm{CO}_{2}$ and remained blue. Under these flow conditions, the large Peclet number, $P e=300$, indicates that the difference in $\mathrm{CO}_{2}$ concentrations between the two streams remains sharp over the whole array.

Temporal control of droplet gas content is obtained by modulating the gas content of the carrier oil, which can be achieved in two different ways. The first is to vary the gas content of the flowing oil prior to entering the chip while keeping the flow rates $Q_{1}$ and $Q_{2}$ constant (Fig. 5C) and the second is to alternate the oil flow rates, $Q_{1}$ and $Q_{2}$ (Fig. 5D). We demonstrate how these two techniques induce oxygenation/deoxygenation cycles of a trapped droplet.

The droplet was monitored for close to an hour as the oxygen content was oscillated from ambient to zero oxygen content with a period of approximately 20 minutes (Fig. 5C). In this case, a single oil inlet was used and the flow was split equally into the two oil channels of the flow focuser $\left(Q_{1}=Q_{2}, C_{1}=C_{2}\right)$. The oxygen concentration of the oil was changed prior to entering the chip using a gas exchange vial described in Section IIB, where the oil equilibrates to the vial's oxygen concentration by flowing through gas permeable tubing. To vary the oxygen content of the oil, the oxygen partial pressure in the vial was changed. The oxygen change in the anchored droplet was slow since it is limited by the equilibration time of the oil in the exchange vial. 
Faster changes are possible by using two independent oil inlets at different oxygen concentrations $\left(C_{1} \approx 0 \mathrm{kPa}\right.$ and $C_{2} \approx 21$ $\mathrm{kPa}$ ) as shown in Fig. 5D. By alternating the oil flow rates $Q_{1}$ and $Q_{2}$, oxygen changes in less than 10 seconds were readily attainable. The flows were modulated by using two different syringe pumps, an operation that could be automated to study the effect of cycling rate on a biological or chemical process. Furthermore, faster oscillations should be attainable by using faster flow rates or potentially by switching to pressure driven control systems.

The operations shown here demonstrate the most basic spatial and temporal control of gas content in droplet arrays. More complex operations, such as continuous gradients of oxygenation for example, can be obtained by combining the techniques described above or by adding more oil inlets with different gas contents. This opens the possibility of using a large array of droplets to perform dynamic assays over a range of conditions.

\section{B. Sickling of red blood cells}

To demonstrate the control and utility of gas exchange on anchored droplets, we show here experiments on the sickling of red blood cells. Sickle cell anemia is a genetic disease due to a single mutation in hemoglobin. ${ }^{25,26}$ After oxygen is released in the tissues, a conformational change in mutant hemoglobin (HbS) exposes a hydrophobic residue on the surface of the protein that provokes its polymerization. This in turn leads to the red blood cells taking on a "sickle" shape, from which the name of the disease derives, and greatly losing their deformability provoking vaso-occlusions. Sickling is reversible and highly dependent on oxygen concentration. Red blood cells are exposed to cycles of oxygen rich and oxygen poor environments in vascular circulation and residual hemoglobin fibers are known to play a role in the dynamics of sickling. ${ }^{27}$ Therefore, it is of biological importance to reproduce these cycles of oxygenation/ deoxygenation to study its effect on red blood cell sickling. Anchored droplet arrays provide a tool to oscillate the oxygen partial pressure while continuously observing a small population of red blood cells.

Polarization microscopy was used to detect intracellular hemoglobin fibers based on their birefringence. ${ }^{28}$ It is compatible with microfluidic approaches and we have recently shown that polarization microscopy can be used to visualize intracellular hemoglobin fibers in red blood cells encapsulated in flowing droplets. ${ }^{17}$ By observing the sample between crossed polarizers, red blood cells with dissolved hemoglobin appear dark while those with hemoglobin fibers rotate the polarization and therefore appear bright relative to the background.

By using flow conditions in the parking mode, droplets with encapsulated sickle red blood cells were trapped in an array of anchors. Two oil inputs were used to obtain a channel with varying oxygen concentration along its width (Fig. 6A). The same chip geometry as in Fig. 5B was used and droplets with sickle red blood cells were trapped in two rows along the length of the channel. The continuous flow of oil $\left(4 \mu \mathrm{L} \min ^{-1}\right)$ was enough to keep the cells in constant motion and suspension in the droplet. The droplets in the upper row have oxygen concentrations close to ambient and the cells appear dark with no indication of intracellular hemoglobin fibers (Fig. 6A, left and Movie $\mathrm{S} 4$ ). In contrast, droplets in the bottom row were deoxygenated and intracellular fibers appear as bright spots within the cells (Fig. 6A, right and Movie S4 ). This spatial control of droplet oxygen content paves the way for experiments on multiple rows of droplets kept under different oxygen partial pressures to determine, for example, the critical oxygen concentration for hemoglobin polymerization.

The flow of deoxygenated oil into a test region containing a linear array of oxygenated droplets is shown in Fig. 6B and Movie S5 . The linear array contains droplets separated by 600 $\mu \mathrm{m}$. Initially, the droplets were oxygenated and the red blood cells inside all the drops appear dark. Deoxygenated oil was then flowed at a rate of $1 \mu \mathrm{L} \mathrm{min}{ }^{-1}$ into the test region replacing the oxygenated oil. The droplets in the linear array are deoxygenated sequentially, with the red blood cells in droplets upstream appearing bright earlier than those downstream. The mean flow velocity was calculated to be $0.15 \mathrm{~mm} \mathrm{~s}^{-1}$ which means that the deoxygenated oil traveled between adjacent droplets in 4 seconds, which is consistent with the time interval for the dark to bright transition in sequential droplets. By controlling the flow rate and droplet spacing one can trigger a reaction in different droplets at different times. In this way, a single snapshot can yield information on the dynamics of a chemical process.

Finally, the oxygen concentration in a single trapped droplet can be oscillated to subject red blood cells to repeated cycles of oxygenation/deoxygenation (Fig. 6C and Movie S6 ). Here the oxygen concentration was oscillated by alternating the flow from two oil inputs containing zero and ambient oxygen, respectively. The flow of oil in the test region is altered at $t=5 \mathrm{~s}$ to deoxygenated oil, at $t=155 \mathrm{~s}$ to oxygenated oil and finally at $t=225 \mathrm{~s}$ back to deoxygenated oil. The sickle red blood cells in the droplets undergo transitions from dark to bright that follow the changes of oxygen content in the oil. We observe a delay of approximately 30 seconds between the alternation of the oil flows $\left(Q_{1}\right.$ and $\left.Q_{2}\right)$ and the dark to bright transitions of the red blood cells. This delay is due to the time required for the oil to travel from the inlet of the test region to the anchored droplet. The low oil flow used in this experiment, $1 \mu \mathrm{L} \mathrm{min}{ }^{-1}$, is insufficient to keep the cells in constant suspension and the cells sediment at later times in the image sequence. Higher oil flows can be used if it is essential to keep cells in constant motion and suspension.

The experiments above demonstrate how anchored droplets can be used to study the effect of repeated sickling and de-sickling events on individual red blood cells. Also, by combining these cycles with spatial control of oxygen content, droplets with oscillating oxygen partial pressures can be viewed simultaneously with control droplets that undergo no oxygen variation. Future work will focus on more closely reproducing the conditions that red blood cells encounter in the vascular system, in particular, with respect to cycling rates, oxygen partial pressure and shear forces. Moreover, anchors and rails can be used to organize droplets of different contents in arrays. This will provide a means to study in a droplet array the effect of potential therapeutics on sickling under conditions that approximate those in the blood.

\section{Summary and conclusion}

In summary, we have demonstrated a robust way to guide and hold drops of a few nanolitres in a 2D area, without relying on the side walls or other structures within the microchannel. This 

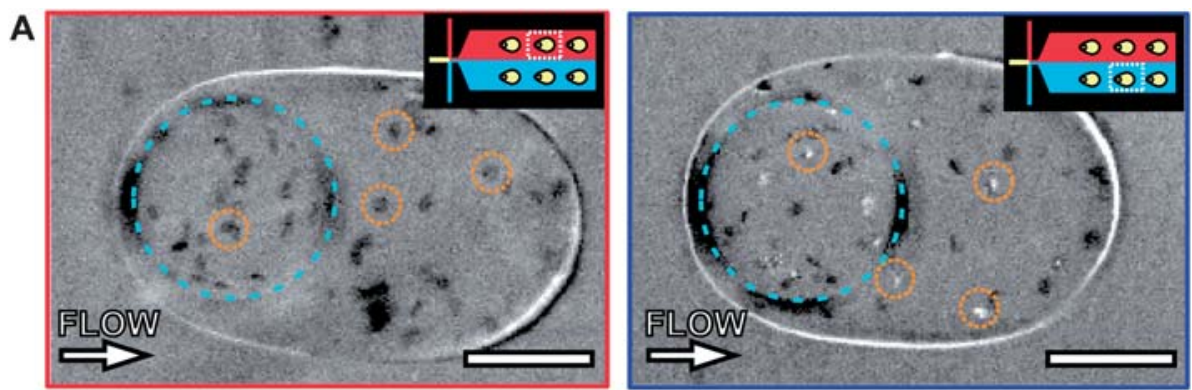

B

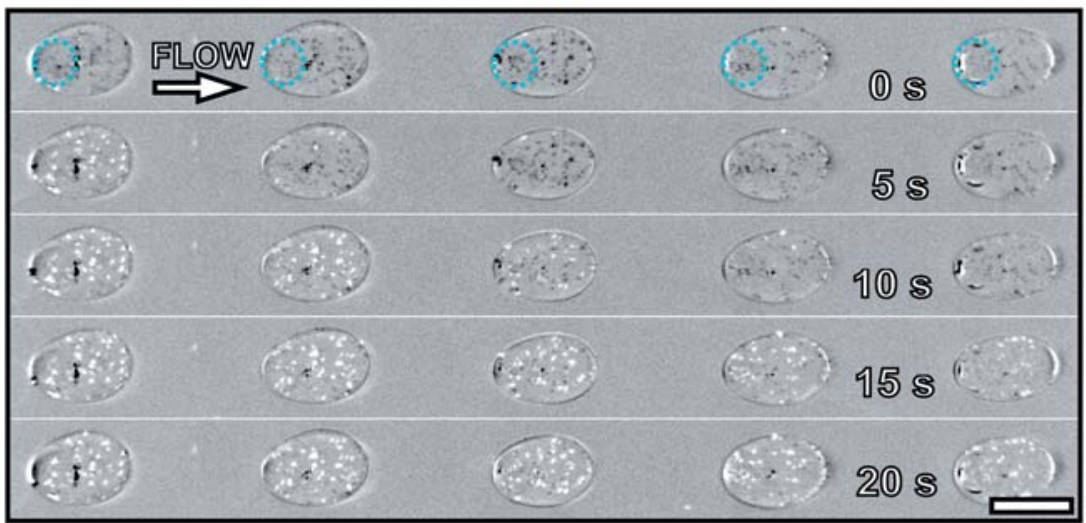

C

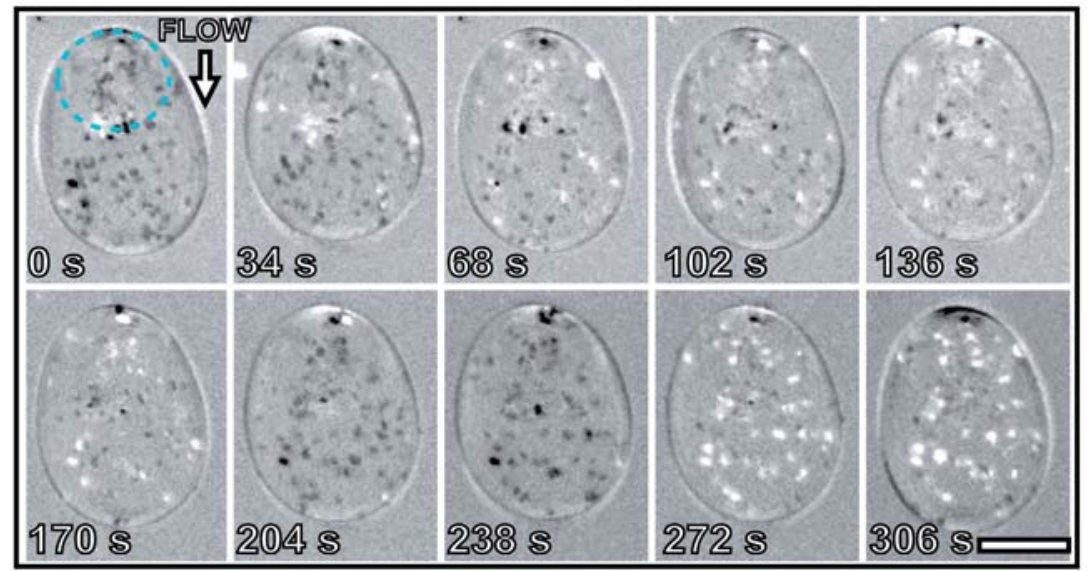

Fig. 6 (A) Simultaneous polarization microscopy images of anchored droplets in a stream of oxygenated oil (left) and deoxygenated oil (right). Images were taken with a $10 \times$ objective and the background was subtracted. A few red blood cells in each image are circled in orange. The contour of the hole is outlined in blue. Using the notation defined in Fig. 5A: $Q_{1}=Q_{2}=2 \mu \mathrm{L} \mathrm{min}{ }^{-1}$ and the partial pressure of oxygen is equal to $C_{1}=21 \mathrm{kPa}$ and $C_{2}=0 \mathrm{kPa}$. The inset shows the droplet position in the channel. The scale bar represents $100 \mu \mathrm{m}$. (B) Polarization microscopy image sequence showing the polymerization of intracellular hemoglobin in a linear array of anchored droplets as deoxygenated oil flows from left to right. Images were taken with a $4 \times$ objective and the background was subtracted. The contour of the hole is outlined in blue in the first image. The oil flow rate is $1 \mu \mathrm{L} \mathrm{min}{ }^{-1}$. The scale bar represents $200 \mu \mathrm{m}$. (C) Polarization microscopy image sequence showing the polymerization and depolymerization of intracellular hemoglobin due to the alternating flows of the two oil channels. Images were taken with a $4 \times$ objective and the background was subtracted. The contour of the hole is outlined in blue in the first image. The partial pressures of oxygen of the oil channels are $C_{1}=21 \mathrm{kPa}$ and $C_{2}=0 \mathrm{kPa}$. The flow changes are the following $t=5 \mathrm{~s}: Q_{1}=1 \rightarrow 0 \mu \mathrm{L} \mathrm{min}{ }^{-1}, Q_{2}=0 \rightarrow 1 \mu \mathrm{L} \mathrm{min}^{-1} ; t=155 \mathrm{~s}: Q_{1}=0 \rightarrow 1 \mu \mathrm{L} \mathrm{min}^{-1}, Q_{2}=1 \rightarrow 0 \mu \mathrm{L} \mathrm{min}{ }^{-1} ; t=225: Q_{1}=1 \rightarrow 0 \mu \mathrm{L} \min ^{-1}, Q_{2}=0 \rightarrow 1$ $\mu \mathrm{L} \mathrm{m^{-1 }}$. The scale bar represents $100 \mu \mathrm{m}$. In all cases, the height $h$ of the anchor is $50 \mu \mathrm{m}$ and its depth $p$ is $50 \mu \mathrm{m}$.

was used to anchor drops subjected to continuous flow of the carrier phase, an important step for producing arrays of drops for lab on a chip applications. The anchor strength determines the maximum flow rate at which a droplet can be trapped. This strength depends on the hole geometry and the droplet size, such that the device can be loaded and emptied by changing the flow rate. Moreover, it can also be run in different modes which allow the observation of drops either sequentially in "buffer mode" or for a long duration in "parking mode". In parking mode, patterning the holes in a grid allows observation of a droplet array. Finally, guiding drops by rails was shown through linear structures of arbitrary shape.

We have also shown how the gas exchange between the flowing oil and the stationary droplet provides a way to modify the drop contents after anchoring. The gas exchange can be used to modify the $\mathrm{pH}$ or oxygen partial pressure within the drops, for example to produce a droplet array with variable conditions over time and space. We used it to selectively provoke intracellular 
hemoglobin polymerization in certain droplets in an array, while temporal control was used to produce cycles of oxygenation and de-oxygenation on encapsulated populations of red blood cells. This spatial and temporal control can now be implemented to study the effect of medical treatments on the polymerization and de-polymerization of intra-cellular hemoglobin, while simulating the cyclic oxygen variations in flowing blood.

This technique should be compared with other approaches that have already been published for arraying drops. In contrast with methods that rely on transport in confined microchannels, ${ }^{12,13,15}$ the rails and anchors provide truly 2D arrays, which greatly reduces the interactions between drops and which allows several inputs to converge on a single test section. Gas control could thus be performed without resorting to a supplementary layer of microchannels. The other two-dimensional approach $^{\mathbf{1 4 , 1 6}}$ produces similar arrays but does not provide a method to selectively place droplets in selected traps. The integration of rails and anchors, possibly augmented with external actuation, would allow such selective positioning. Finally, all of these advances in arraying techniques should yield equivalent capabilities as drop deposition on a flat surface, ${ }^{29-31}$ while keeping the flexibility and functionality of droplet microfluidics.

\section{Acknowledgements}

Special thanks to François Lionnet for providing blood from sickle cell patients and Jean-Christophe Baret for providing the perfluorinated surfactant used in our studies. We would also like to thank Pierre-Louis Tharaux, Ashleigh Theberge and Samir Zard for helpful discussions. The authors also thank Caroline Frot for microfabrication assistance. This project was partially supported by Region Ile-de-France Nanosciences Competence Center and the CNRS Interdisciplinary Program "Interface Physique Chimie Biologie: soutien à la prise de risque”. P.A. acknowledges the financial support from CNRS and Ecole Polytechnique.

\section{References}

1 G. Ramsay, Nat. Biotechnol., 1998, 16, 40-44.

2 K. Appasani, Bioarrays: from Basics to Diagnostics, Humana Pr Inc, 2007.

3 T. Thorsen, S. Maerkl and S. Quake, Science, 2002, 298, 580.
4 I. Meyvantsson, J. W. Warrick, S. Hayes, A. Skoien and D. J. Beebe, Lab Chip, 2008, 8, 717-724.

5 M. Chabert, K. D. Dorfman and J. L. Viovy, Electrophoresis, 2005, 26, 3706-3715.

6 G. Cristobal, J. P. Benoit, M. Joanicot and A. Ajdari, Appl. Phys. Lett., 2006, 89, 034104.

7 K. Ahn, J. Agresti, H. Chong, M. Marquez and D. A. Weitz, Appl. Phys. Lett., 2006, 88, 264105.

8 C. N. Baroud, M. R. de Saint Vincent and J.-P. Delville, Lab Chip, 2007, 7, 1029-1033.

9 E. Brouzes, M. Medkova, N. Savenelli, D. Marran, M. Twardowski, J. Hutchison, J. Rothberg, D. Link, N. Perrimon and M. Samuels, Proc. Natl. Acad. Sci. U. S. A., 2009, 106, 14195.

10 M. L. Cordero, D. R. Burnham, C. N. Baroud and D. McGloin, Appl. Phys. Lett., 2008, 93, 034107.

11 E. Verneuil, M. L. Cordero, F. Gallaire and C. N. Baroud, Langmuir, 2009, 25, 5127-5134.

12 J. Shim, G. Cristobal, D. R. Link, T. Thorsen, Y. Jia, K. Piattelli and S. Fraden, J. Am. Chem. Soc., 2007, 129, 8825-8835.

13 W. Shi, J. Qin, N. Ye and B. Lin, Lab Chip, 2008, 8, 1432-1435.

14 A. Huebner, D. Bratton, G. Whyte, M. Yang, A. Demello, C. Abell and F. Hollfelder, Lab Chip, 2009, 9, 692.

15 C. Schmitz, A. Rowat, S. Köster and D. Weitz, Lab Chip, 2009, 9, 44 49.

16 Y. Bai, X. He, D. Liu, S. Patil, D. Bratton, A. Huebner, F. Hollfelder, C. Abell and W. Huck, Lab Chip, 2010, 10, 1281-1285.

17 P. Abbyad, P. Tharaux, J. Martin, C. Baroud and A. Alexandrou, Lab Chip, 2010, 10, 2505-2512.

18 K. Stephan, P. Pittet, L. Renaud, P. Kleimann, P. Morin, N. Ouaini and R. Ferrigno, J. Micromech. Microeng., 2007, 17, N69.

19 D. Bartolo, G. Degré, P. Nghe and V. Studer, Lab Chip, 2008, 8, 274 279.

20 J. Clausell-Tormos, D. Lieber, J. C. Baret, A. El-Harrak, O. J. Miller, L. Frenz, J. Blouwolff, K. J. Humphry, S. Köster, H. Duan, C. Holze, D. A. Weitz, A. D. Griffiths and C. A. Merten, Chem. Biol., 2008, 15, 427-437.

21 H. Hele-Shaw, Nature, 1898, 58, 520.

22 S. L. Anna, N. Bontoux and H. A. Stone, Appl. Phys. Lett., 2003, 82, 364-366.

23 J. Bush, J. Fluid Mech., 1997, 352, 283-303.

24 K. Ahn, C. Kerbage, T. Hynt, R. M. Westervelt, D. R. Link and D. A. Weitz, Appl. Phys. Lett., 2006, 88, 024104.

25 G. Serjeant, Lancet, 1997, 350, 725-730.

26 M. Stuart and R. Nagel, Lancet, 2004, 364, 1343-1360.

27 W. Eaton and J. Hofrichter, Blood, 1987, 70, 1245-1266.

28 H. Sunshine, J. Hofrichter, F. Ferrone and W. Eaton, J. Mol. Biol., 1982, 158, 251-273.

29 F. Lemaire, C. Mandon, J. Reboud, A. Papine, J. Angulo, H. Pointu, C. Diaz-Latoud, C. Lajaunie, F. Chatelain and A. Arrigo, PLoS One, 2007, 2, e163.

30 H. Kim, S. Vishniakou and G. W. Faris, Lab Chip, 2009, 9, 1230 1235 .

31 L. Mugherli, O. Burchak, L. Balakieva, A. Thomas, F. Chatelain and M. Balakirev, Angew. Chem., Int. Ed., 2009, 48, 7639-7644. 